\title{
Lévy night flights by the jellyfish Periphylla periphylla
}

\author{
Karl I. Ugland ${ }^{1}$, Dag L. Aksnes ${ }^{2}$, Thor A. Klevjer ${ }^{1,4}{ }^{,}$Josefin Titelman $^{1}$, \\ Stein Kaartvedt ${ }^{1,3 *}$ \\ ${ }^{1}$ Department of Biosciences, University of Oslo, Pb. 1066 Blindern, 0316 Oslo, Norway \\ ${ }^{2}$ Department of Biology, University of Bergen, 5020 Bergen, Norway \\ ${ }^{3}$ King Abdullah University of Science and Technology, Red Sea Research Center, Thuwal 23955-6900, Saudi Arabia \\ ${ }^{4}$ Present address: Institute of Marine Research, PO Box 1870 Nordnes, 5817 Bergen, Norway
}

\begin{abstract}
Jellyfish blooms occur in marine environments around the world and have been linked to over-fishing, eutrophication and climatic change. In some coastal areas of Norway, the circumglobal Periphylla periphylla has increased to exceptionally high abundances and has replaced fish as the main planktivorous predator despite the ineffectiveness of its non-visual predation compared to visual fish predation. Using data from a bottom-mounted acoustic platform, we collected 12341 in situ measurements of individual vertical movements of large individuals of $P$. periphylla. These jellyfish are characterized by a stepwise vertical movement. The distribution of their vertical swimming distances was extremely left skewed; about $85 \%$ of the swimming distances were less than $3 \mathrm{~m}$, and a few displacements were extremely long with a maximum of $85 \mathrm{~m}$. Chi-square tests of goodness of fit to the tail and Akaike's information criterion gave overwhelming evidence of the truncated power law. There was a clear diel pattern in the exponent with values significantly larger than 3 during the daytime and significantly lower than 3 at night. This pattern means that $P$. periphylla switches from relatively limited movements during the day to Lévy-like flights during the night. Since the abundance of zooplankton is large in the P. periphylla fjord, Brownian motion, rather than Lévy flight, is predicted by the optimal foraging hypothesis. It is therefore possible that the Lévy-like search pattern has evolved in the food-scarce oceanic environment, which is the main natural habitat of P. periphylla. Alternatively, the large individuals of the population addressed here may forage on scarcer prey sources than the main prevailing zooplankton in Lurefjorden.
\end{abstract}

KEY WORDS: Jellyfish $\cdot$ Swimming patterns $\cdot$ Diel cycle $\cdot$ Truncated power law $\cdot$ Lévy flight Acoustics

Resale or republication not permitted without written consent of the publisher

\section{INTRODUCTION}

Available evidence suggests that jellyfish blooms are increasing globally in response to humaninduced stresses (Mills 2001, Purcell et al. 2007, Richardson et al. 2009). In some Norwegian fjords the deep-sea jellyfish Periphylla periphylla has reached densities several orders of magnitude higher than in the open sea (Fosså 1992, Dalpadado et al. 1998,
Sørnes et al. 2007). A common feature of these 'jellyfish fjords' is that in contrast to adjacent fjords, the abundance of fish is very low. In order to identify the factors leading to such dramatic changes in coastal ecosystems, it is essential to take into account the different search strategies of fish and jellyfish.

Compared to visual predators such as fish, the tactile predator $P$. periphylla is inefficient (Sørnes \& Aksnes 2004, Sørnes et al. 2008). While a fish may 
spot a zooplankton prey at some distance away from its own body, prey detection in tactile predators like P. periphylla requires close contact between its body surface and the prey. In an enclosed area, like a fjord, a low predation efficiency of an apex predator should lead to a high density of prey organisms. This effect is precisely what is observed in the well-studied Lurefjorden, where unusually high abundances of zooplankton co-occur with the exceptionally high abundance of $P$. periphylla and low abundances of fish (Eiane et al. 1999, Bagøien et al. 2001). Since this 'jellyfish fjord' has high densities of crustacean zooplankton and visually foraging competitors are practically absent, the fjord provides a unique opportunity to study the non-visual foraging strategy of jellyfish.

Kaartvedt et al. (2011) reported a diverse behavioural repertoire of $P$. periphylla that extends far beyond a general diel vertical migration (DVM) of the average population. Data obtained using a bottom-mounted echo sounder in Lurefjorden revealed that the population is divided into groups with different behaviours. Individuals of behavioural Mode 1 (sensu Kaartvedt et al. 2011) perform synchronized DVM in the upper $\sim 100 \mathrm{~m}$ above an acoustic-scattering layer of krill. Members of Mode 2 stay beneath the krill layer during the day $(\sim 160$ to $200 \mathrm{~m}$ ) and synchronously ascend towards the surface in the evening and descend in the morning. Mode 3 is characterized by continuous vertical swimming between $\sim 200 \mathrm{~m}$ and the bottom during the day and an approximately uniform distribution in the entire water column during the night. Finally, a group of larger individuals (size derived from the acoustic backscatter) forms Mode 4 with a main distribution between $\sim 130 \mathrm{~m}$ and the bottom. These jellyfish shift between remaining almost motionless and relocating in vertical steps during both the day and night.

These 4 behavioural modes of $P$. periphylla were easily distinguished throughout the whole investigation period of several months (Kaartvedt et al. 2011). The behavioural patterns deviate significantly from a synchronous daily mass movement of the entire population. In particular, the behaviour of the larger individuals (Mode 4) involves step-wise vertical bouts (Kaartvedt et al. 2007, Klevjer et al. 2009). Several factors may influence the observed pattern of movement: (1) predation on motile prey like krill or nonmotile prey like dormant Calanus (Youngbluth \& Båmstedt 2001, Sørnes et al. 2008), (2) increasing mate encounter rates (Tiemann et al. 2009), and (3) avoidance of light above a threshold intensity (Jarms et al. 2002, Dupont et al. 2009). Also, the internal state may influence vertical movements, and individual variation in DVM among zooplankton has been ascribed to body condition (Hays et al. 2001).

A characteristic feature of stochastic environments is that the predators have relatively little information about the distribution of their prey. Rather than using knowledge of last site of encounter, the optimal search becomes Lévy flights (Viswanathan et al. 1999). In contrast to Brownian motion, where the distance covered by the predator is proportional to the logarithm of time, Lévy flights induce much longer displacements over shorter time periods because the step lengths have a distribution where the frequency of extreme events cannot be neglected. Thus, while most steps are short, some step lengths are unusually long, and the histogram of the step lengths will have a characteristic heavy tail approximating a power law with an exponent between 1 and 3 . The benefit is an efficient exploitation of prey or mates nearby, combined with a means to discover resources further away. Realization of Lévy flights has been suggested in many biological systems (Sims et al. 2008) and is generally believed to be the best strategy for optimizing success when performing a random search for a target with a patchy distribution (Viswanathan et al. 1999, Bartumeus et al. 2002).

In this study, we wanted to investigate whether the data obtained by in situ measurements of individual movement provides support for concluding whether P. periphylla performs a Lévy-like flight or a Brownian random walk, that is, whether the distribution of the step lengths has a tail approximating a power law (scale-free motion) or a tail with negligible probability. For this assessment, we analysed a large data set of more than 12000 step lengths. Since a pure Lévy flight is based on a distribution with infinite variance, it cannot be realized in nature because there are natural upper boundaries for the length of any movement. For example, a vertical movement of a jellyfish must necessarily be shorter than the height of the water column. We therefore investigated the performance of 3 statistical distributions, (1) the power law, (2) the truncated power law and (3) the exponential model.

\section{MATERIALS AND METHODS}

We tested the universality of Lévy-like flights on a large data set obtained from an investigation of the vertical swimming behaviour of Periphylla periphylla. Using a bottom-mounted acoustic platform, a 
total of 12341 in situ measurements of individual vertical movements were made. In order to select the model that is best supported by the data, we use the recommended 'model selection' procedure (Johnson \& Omland 2004, Edwards et al. 2007). If the power law or the truncated power law obtain the greatest relative support (Akaike weights), we conclude that the movement is a Lévy-like flight.

\section{Acoustic data}

We used a submerged SIMRAD EK60 echo sounder to follow the vertical movements and step lengths of individuals. The echo sounder was placed on the seabed at a depth of $280 \mathrm{~m}$, with a $38 \mathrm{kHz}\left(7.1^{\circ}\right.$ beam width) transducer in upward-looking mode, scanning the water column once per second. The transceiver was housed in a pressure-proof glass sphere next to a pressure-proof transducer. The transducer was mounted in a steel frame with gimbal couplings to ensure horizontal orientation of the transducer surface. The echo sounder was connected to shore by a cable for power and transmission of digitized data to a computer.

Our data were sampled during $3 \mathrm{wk}$ in January 2007 and analysed for a $150 \mathrm{~m}$ depth range above the transducer. At the applied frequency of $38 \mathrm{kHz}$, individual acoustic targets in the fishless, deep waters of Lurefjorden can be ascribed to $P$. periphylla (Kaartvedt et al. 2007, 2011). We used a single echo detection threshold of $-70 \mathrm{~dB}$. This threshold eliminated the smaller jellyfish from the data set such that our conclusions refer to the larger specimens of the population only. The echoes from individual targets were usually considerably stronger than this lower threshold, and the analysed acoustic segments (see next paragraph) had a median target strength (TS) of $-58.3 \mathrm{~dB}$, with quartiles of -60.3 and $-56.7 \mathrm{~dB}$.

The procedure of combining sequential echoes from the same target into 'tracks' describing the swimming paths of individuals is known as target tracking (Brede et al. 1990). The vertical swimming behaviour of $P$. periphylla was analysed with specially designed software for target tracking (Balk \& Lindem 2000). Due to weak currents in the enclosed fjord basin, individual jellyfish remain in the acoustic beam for extended periods (e.g. see Fig. 1), making the conditions ideal for acoustic target tracking of individuals. The swimming paths of $P$. periphylla were split into segments according to vertical behaviour. Each segment consisted of either upwards or downwards swimming, or no vertical motion, defined as vertical speeds $<1 \mathrm{~cm} \mathrm{~s}^{-1}$. Distributions were based on the step lengths of such segments.

We analysed the data by subdividing the observations in 2 different ways. First the data were classified into day or night, where all tracks measured between 08:00 and 16:00 h UTC were recognized as day and the remaining tracks as night. This procedure gave 6937 vertical step lengths during the day and 5404 vertical step lengths during the night. Secondly, we aggregated all the measured tracks into 12 subsets consisting of time intervals of $2 \mathrm{~h}$ starting from midnight. Thus tracks measured between 00:00 and $02: 00 \mathrm{~h}$ UTC belong to the interval with midpoint 01:00 h. This results in 12 midpoints to which each track was ascribed based on the nearest midpoint.

Since the acoustic beam expands by range and therefore potentially also the probability of detecting longer steps, we checked if there were systematic differences in the range of targets between day and night. No evident changes were found, with median ranges being $115 \mathrm{~m}$ (quartiles of 105 and $127 \mathrm{~m}$ ) and $111 \mathrm{~m}$ (quartiles of 79 and $130 \mathrm{~m}$ ) at day and night, respectively.

\section{Data analysis}

Considering the size of individuals and the vertical resolution of measurements of the echo sounder, we decided to only include movements exceeding $1 \mathrm{~m}$. With a minimum step length of $x_{\min }$ the density of the power law is (when studying the shape of the tail we need to consider larger minimum values than $x_{\min }=$ $1 \mathrm{~m})$ :

$$
f_{\text {power }}(x)=(\alpha-1)\left(x_{\min }\right)^{-1}\left(\frac{x_{\min }}{x}\right)^{\alpha} \text { for } x \geq x_{\min }
$$

The asymptotic variance of the maximum likelihood estimator (MLE) of the single parameter $\alpha$ is given by the formula $(\alpha-1)^{2} / n$ (Clauset et al. 2007). Since the number of observations $(n)$ is of the order of several hundreds, the $95 \%$ confidence interval may be approximated by $\hat{\alpha} \pm 2 \frac{\hat{\alpha}-1}{\sqrt{n}}$.

To account for the fact that any step length in nature has an upper bound $\left(x_{\max }\right)$, we also approximated the observed step lengths by a truncated power law, which has the density:

$$
\begin{gathered}
f_{\text {power truncated }}(x)=(\alpha-1)\left(x_{\min }\right)^{-1}\left(\frac{x_{\min }}{x}\right)^{\alpha} / \\
\left(1-\left(x_{\min } / x_{\max }\right)^{\alpha-1}\right) \text { for } x_{\min } \leq x \leq x_{\max }
\end{gathered}
$$

The standard alternative to these 2 types of power distributions with non-negligible large step lengths 
is the exponential distribution, which has a finite variance with small probabilities of step lengths far from the average value (exponentially declining with increasing length):

$$
f_{\text {exponential }}(x)=\lambda \exp \left(-\lambda\left(x-x_{\min }\right)\right) \text { for } x \geq x_{\min }
$$

To test whether the data provide more evidence for a Lévy-like flight or a Brownian random walk, we used the recommended theory of model selection based on Akaike's information criterion (AIC) (Edwards 2008). We followed the procedure and equations given by Johnson \& Omland (2004) and Edwards et al. (2007, their Box 1).

Assessment of the fit of the power law to the data was performed with the Pearson chi-square test:

$$
\chi^{2}=\sum_{k=1}^{m} \frac{\left(O_{k}-E_{k}\right)^{2}}{E_{k}}
$$

where $O_{k}$ and $E_{k}$ are the observed and expected number of step lengths in the $k^{\text {th }}$ class for $k=1, \ldots, m$. Here $m$ is the number of considered step length intervals (i.e. the number of classes), so the degree of freedom is $\mathrm{df}=m-1-p$, where $p$ is the number of parameters in the model which are estimated from the data. Since $x_{\min }$ is predefined rather than estimated from the data, it is not included in the df calculation. Thus, the value of $p$ is 1 for the power law $(\alpha), 2$ for the truncated power law ( $\alpha$ and $x_{\max }$ ) and 1 for the exponential model $(\lambda)$. This approach gives the following df: $\mathrm{df}$ (power) $=m-1-1=m-2$, df(power truncated $)=m-1-2=m-3$ and $\operatorname{df}($ exponential $)=$ $m-1-1=m-2$. In order to keep the number of expected observations in each class above 5, the step length intervals were increased in a geometric series according to an increment of $30 \%$, i.e. the end points of the step length intervals were chosen as 1.0, 1.3, $1.7,2.2,2.9,3.7,4.8,6.3,8.2,10.6,13.8,17.9,23.3$, $30.3,39.4,51.2,66.5$ and 86.5 . There are 2 advantages of using these 17 intervals, 1.0-1.3, 1.3-1.7, ..., 66.5-86.5: First, they apply to all the investigated subsets of the data, because they tend to incorporate more than 5 observations in the intervals. Second, the power law becomes a straight line when the logarithm of the tail, $\log \left(1-F_{\text {power }}(x)\right)$, where $F$ is the cumulative probability function of the step length,is plotted against the logarithm of the step length, $\log (x)$. In order to get positive values on the ordinate it is common to plot the logarithm of the ranks (where the longest step length is given Rank 1) against the logarithm of the step length. This rank-frequency plot is useful to detect any deviation from the power law. An interval increment of $30 \%$ could be applied to all our analyses when looking for deviations from a straight line in the logarithmic plot of ranks versus step length.

Performance of a Lévy-like flight depends on the ability to undertake some long movement step lengths according to a power law or truncated power law. Therefore, the essence of Lévy-like flights lies in the tail of the distribution governing the length of the steps. In other words, the distribution of the step lengths may very well be described by 2 widely different distributions: one for the small and another for the long steps. More precisely, the tail of a Lévy distribution may be approximated by a power law with an exponent between 1 and 3. Application of this asymptotic property to real data requires a specification of what should be considered as the tail. Two factors are important when defining the step length intervals of the tail. First, these intervals must contain enough observations for performing ordinary chisquare tests. Second, it is preferable to use the same definition for all the considered subsets (day, night, time of the day). Since the first 4 step length intervals tend to contribute largely to the chi-square value, we decided to define $2.9 \mathrm{~m}$ as the lower limit for the tail of the step length distribution of $P$. periphylla in Lurefjorden. The last $17-4=13$ intervals are considered as the tail. For the $4 \mathrm{~h}$ during the day (09:00, 11:00, 13:00, and 15:00 h UTC) the tail consisted of 7 to $9 \%$ of all the observations, and for the other hours during the night the percentage fluctuated between $14 \%$ (19:00 h UTC) and 29\% (05:00 h UTC).

\section{RESULTS}

During both the day and night, the distribution of the step lengths were extremely left-skewed, with $93 \%$ (day) and $79 \%$ (night) of the movements less than $2.9 \mathrm{~m}$. During the day, only $1.5 \%$ of the step lengths were longer than $6 \mathrm{~m}$, and $0.1 \%$ of the swimming distances exceeded $20 \mathrm{~m}$. During the night, the activity of Periphylla periphylla increased considerably. About $3 \%$ of the step lengths were longer than $16 \mathrm{~m}$, and $0.2 \%$ of the movements constituted extreme distances over $50 \mathrm{~m}$. Fig. 1 shows a vertical step length of $85 \mathrm{~m}$ (as well as examples of individuals with much shorter steps).

We employed MLE to fit the power law, the truncated power law and exponential models to the aggregated day and night data (Fig. 2). Visual inspection shows that the observed step-length frequency distributions may only be described by the power law and the truncated power law. For both day and night the exponents of the 2 power laws were 

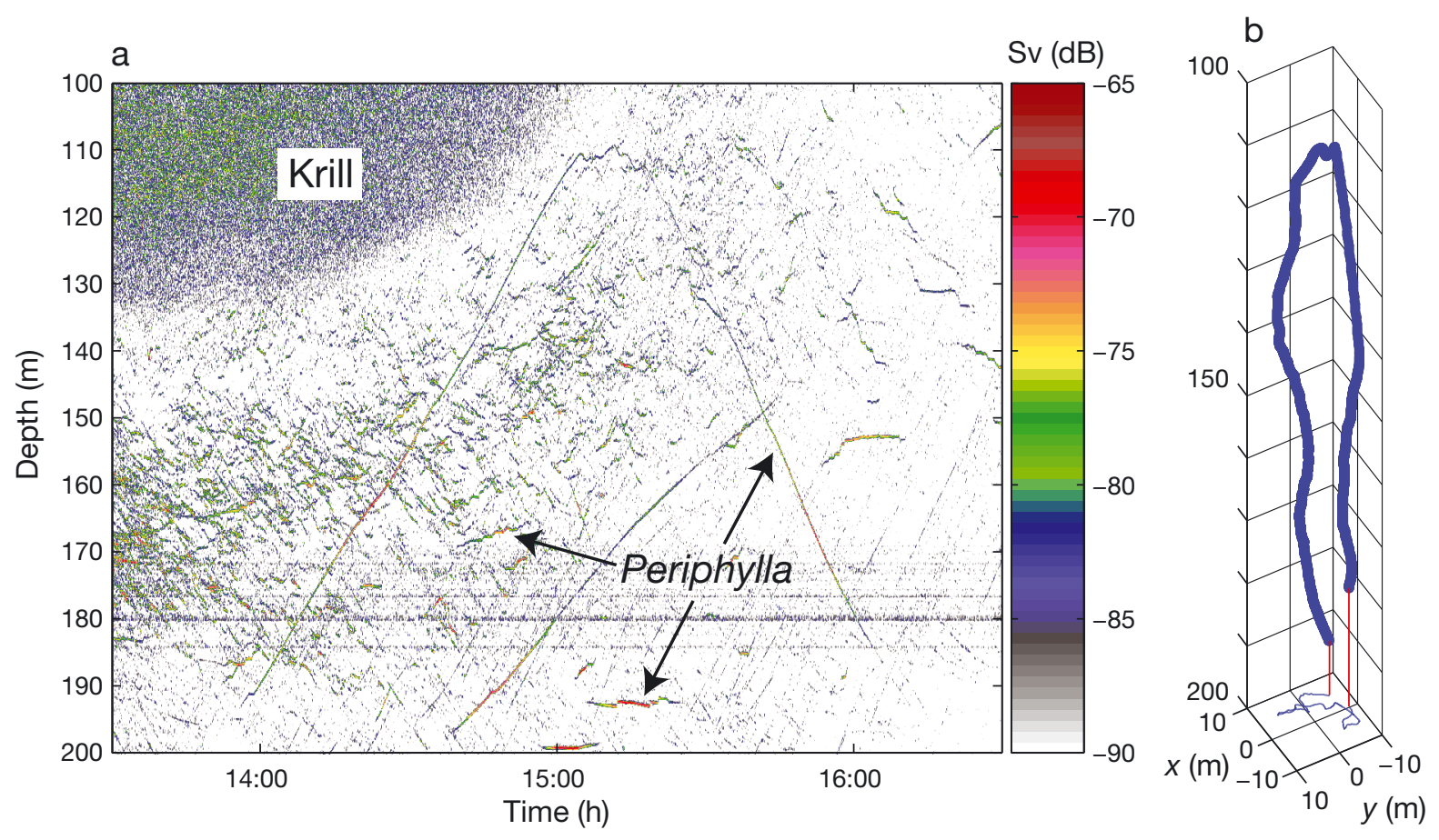

Fig. 1. Normal and extreme vertical movements of Periphylla periphylla. (a) In the echogram only the $y$-axis represents jellyfish displacement, while echo traces along the $x$ axis represent progressing time. Since a single organism remains in the acoustic beam for many successive records, the acoustic returns will be visible as a continuous trace on the echogram. One individual (mean target strength $-54 \mathrm{~dB}$ ) ascending $85 \mathrm{~m}$ and subsequently descending is recorded for $\sim 2 \mathrm{~h}$. (b) The 3-dimensional movement of this individual based on smoothed data (linear interpolation) from acoustic target tracking is depicted. Colour scale refers to backscattering strength $(\mathrm{Sv})$ values $(\mathrm{dB})$ for the echogram

practically equal (Table 1): 3.4 (day) and 2.4 (night), and the AIC weights (wAIC) strongly favoured the power laws. Since an exponent value of 3.4 is not within the Lévy range of 1 to 3 , these $P$. periphylla apparently switch behaviour between restricted random walks during the day and Lévy-like flights during the night. However, binning the step lengths in intervals starting with $1 \mathrm{~m}$ and increasing geometrically with $30 \%$, the observed frequencies differed significantly from the expected frequencies based on both the power law and the truncated power law (chi-square test, Table 1). Keeping in mind that the properties of Lévy flights are revealed in the tail of

Fig. 2. Rank-frequency plots of the move-step length distribution $(\diamond$; mostly appearing as a thick line due to a large number of measurements) for (a) day ( $\mathrm{n}=6937$ steps) and (b) night ( $\mathrm{n}=5404$ steps) with model fits (estimated by maximum likelihood method) of the power law (Power), the truncated power law (Trunc) and the exponential model (Exp). Akaike weights support the 2 power laws and exclude the exponential model (Table 1)
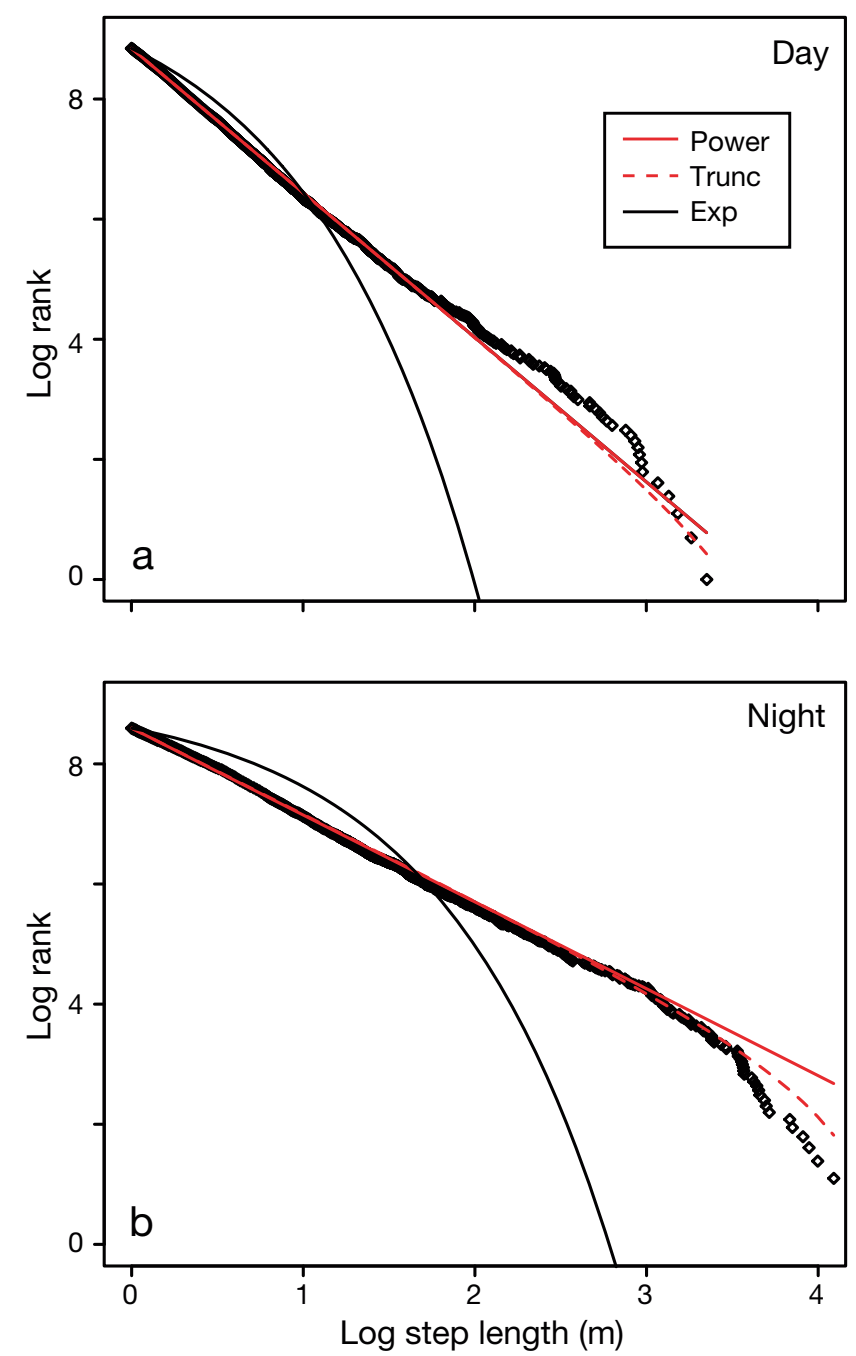
Table 1. Model selection and chi-square test for goodness of fit of the 3 competing models: (1) power law, (2) truncated power law, and (3) exponential model. $p$ : number of parameters estimated from the data with the maximum likelihood method, Exponent: the exponential parameter of the model, wAIC: relative weight of evidence for the model based on Akaike's information criterion (AIC), df: degrees of freedom in the chi-square test, and p: probability of obtaining a deviation from the model as large as the observed value. The 3 models are compared on 4 data sets: Day ( $n=6937$ samples during the day), Night ( $\mathrm{n}=5404$ step lengths during the night), Day tail ( $\mathrm{n}=517$ step lengths $>2.9 \mathrm{~m}$ during the day), Night tail $(\mathrm{n}=1141$ step lengths $>2.9 \mathrm{~m}$ during the night)

\begin{tabular}{|lcccrc|}
\hline & $p$ & Exponent & wAIC & df & $p$ \\
\hline Day & & & & & \\
Power & 1 & 3.41 & 0.58 & 10 & 0.02 \\
Power trunk & 2 & 3.40 & 0.42 & 9 & 0.01 \\
Exponential & 1 & 1.39 & 0.00 & 5 & 0.00 \\
Night & & & & & \\
Power & 1 & 2.45 & 0.00 & 14 & 0.00 \\
Power trunk & 2 & 2.43 & 1.00 & 13 & 0.00 \\
Exponential & 1 & 0.57 & 0.00 & 8 & 0.00 \\
Day tail & & & & & \\
Power & 1 & 3.21 & 0.40 & 6 & 0.31 \\
Power trunk & 2 & 3.18 & 0.60 & 5 & 0.17 \\
Exponential & 1 & 1.39 & 0.00 & 5 & 0.00 \\
Night tail & & & & & \\
Power & 1 & 2.54 & 0.00 & 10 & 0.03 \\
Power trunk & 2 & 2.49 & 1.00 & 9 & 0.15 \\
Exponential & 1 & 0.23 & 0.00 & 7 & 0.00 \\
\hline
\end{tabular}

the distribution, we repeated the analysis for the tail, i.e. for all movements larger than $2.9 \mathrm{~m}$ (which is the end point of the $4^{\text {th }}$ interval). Both power laws fitted the tail of the distribution observed during the day, but only the truncated power law fitted the tail of the night data (Table 1). Thus, the chi-square test for the goodness of fit at the tail and the model selection based on the AIC favours the truncated power law. The observed move-step distributions are consistent with a truncated power law having an exponent that switches between values above and below the critical value 3 . Thus, the data provide evidence of changing movement patterns from day to night.

To further explore whether there is a behavioural pattern over a $24 \mathrm{~h}$ cycle, we subdivided the data into 12 subsets consisting of $2 \mathrm{~h}$ starting at midnight: 00:00-02:00, 02:00-04:00, 04:00-06:00 h, etc. These intervals are denoted by the midpoint hour of the interval, i.e. 01, 03, 05, etc. We repeated the same detailed analysis performed above for each of the 12 subsets (Tables $2 \& 3$ ).

First we notice that the AIC always strongly disfavours the exponential model (Table 2) and the chi-
Table 2. Model selection and chi-square test for goodness of fit of the 3 competing models: (1) power law, (2) truncated power law, and (3) exponential model applied to the 12 subsets of $2 \mathrm{~h}$ intervals around (number of step lengths in parentheses): 1 (734), 3 (650), 5 (698), 7 (991), 9 (2015), 11 (1653), 13 (1726), 15 (1543), 17 (552), 19 (597), 21 (604), 23 (578). Abbreviations as in Table 1

\begin{tabular}{|c|c|c|c|c|c|}
\hline & $p$ & Exponent & wAIC & df & $\mathrm{p}$ \\
\hline \multicolumn{6}{|l|}{ Night 1} \\
\hline Power & 1 & 2.38 & 0.21 & 11 & 0.09 \\
\hline Power trunk & 2 & 2.36 & 0.79 & 10 & 0.03 \\
\hline Exponential & 1 & 0.49 & 0.00 & 8 & 0.00 \\
\hline \multicolumn{6}{|l|}{ Night 3} \\
\hline Power & 1 & 2.35 & 0.08 & 11 & 0.11 \\
\hline Power trunk & 2 & 2.31 & 0.92 & 10 & 0.21 \\
\hline Exponential & 1 & 0.52 & 0.00 & 8 & 0.00 \\
\hline \multicolumn{6}{|l|}{ Night 5} \\
\hline Power & 1 & 2.24 & 0.00 & 11 & 0.03 \\
\hline Power trunk & 2 & 2.17 & 1.00 & 10 & 0.18 \\
\hline Exponential & 1 & 0.45 & 0.00 & 8 & 0.00 \\
\hline \multicolumn{6}{|l|}{ Night 7} \\
\hline Power & 1 & 2.64 & 0.49 & 10 & 0.03 \\
\hline Power trunk & 2 & 2.62 & 0.51 & 9 & 0.01 \\
\hline Exponential & 1 & 0.68 & 0.00 & 7 & 0.00 \\
\hline \multicolumn{6}{|l|}{ Day 9} \\
\hline Power & 1 & 3.45 & 0.42 & 8 & 0.11 \\
\hline Power trunk & 2 & 3.44 & 0.58 & 7 & 0.07 \\
\hline Exponential & 1 & 1.52 & 0.00 & 5 & 0.00 \\
\hline \multicolumn{6}{|l|}{ Day 11} \\
\hline Power & 1 & 3.49 & 0.61 & 7 & 0.02 \\
\hline Power trunk & 2 & 3.48 & 0.39 & 6 & 0.01 \\
\hline Exponential & 1 & 1.47 & 0.00 & 5 & 0.00 \\
\hline \multicolumn{6}{|l|}{ Day 13} \\
\hline Power & 1 & 3.48 & 0.56 & 7 & 0.73 \\
\hline Power trunk & 2 & 3.47 & 0.44 & 6 & 0.54 \\
\hline Exponential & 1 & 1.47 & 0.00 & 5 & 0.00 \\
\hline \multicolumn{6}{|l|}{ Day 15} \\
\hline Power & 1 & 3.21 & 0.66 & 8 & 0.14 \\
\hline Power trunk & 2 & 3.20 & 0.34 & 7 & 0.08 \\
\hline Exponential & 1 & 1.17 & 0.00 & 5 & 0.00 \\
\hline \multicolumn{6}{|l|}{ Day 17} \\
\hline Power & 1 & 2.76 & 0.65 & 8 & 0.37 \\
\hline Power trunk & 2 & 2.75 & 0.35 & 7 & 0.26 \\
\hline Exponential & 1 & 0.79 & 0.00 & 6 & 0.00 \\
\hline \multicolumn{6}{|l|}{ Night 19} \\
\hline Power & 1 & 2.58 & 0.28 & 9 & 0.02 \\
\hline Power trunk & 2 & 2.55 & 0.72 & 8 & 0.02 \\
\hline Exponential & 1 & 0.72 & 0.00 & 6 & 0.00 \\
\hline \multicolumn{6}{|l|}{ Night 21} \\
\hline Power & 1 & 2.36 & 0.38 & 11 & 0.06 \\
\hline Power trunk & 2 & 2.34 & 0.62 & 10 & 0.03 \\
\hline Exponential & 1 & 0.47 & 0.00 & 9 & 0.00 \\
\hline \multicolumn{6}{|l|}{ Night 23} \\
\hline Power & 1 & 2.38 & 0.09 & 11 & 0.00 \\
\hline Power trunk & 2 & 2.34 & 0.91 & 10 & 0.00 \\
\hline Exponential & 1 & 0.60 & 0.00 & 7 & 0.00 \\
\hline
\end{tabular}


Table 3. Model selection and chi-square test for goodness of fit of the 2 competing models: (1) power law and (2) truncated power law applied to the tail (step lengths longer than $2.9 \mathrm{~m}$ ) of the 12 subsets of $2 \mathrm{~h}$ intervals around (number of step lengths in parentheses): 1 (734), 3 (650), 5 (698), 7 (991), 9 (2015), 11 (1653), 13 (1726), 15 (1543), 17 (552), 19 (597), 21 (604), 23 (578). Abbreviations as in Table 1

\begin{tabular}{|lccccc|}
\hline & $p$ & Exponent & wAIC & df & $\mathrm{p}$ \\
\hline Night tail 1 & & & & & \\
Power & 1 & 2.47 & 0.31 & 6 & 0.56 \\
Power trunk & 2 & 2.39 & 0.69 & 5 & 0.42 \\
Night tail 3 & & & & & \\
Power & 1 & 2.46 & 0.15 & 7 & 0.17 \\
Power trunk & 2 & 2.33 & 0.85 & 6 & 0.34 \\
Night tail 5 & & & & & \\
Power & 1 & 2.40 & 0.00 & 7 & 0.19 \\
Power trunk & 2 & 2.20 & 1.00 & 6 & 0.59 \\
Night tail 7 & & & & & \\
Power & 1 & 2.60 & 0.47 & 6 & 0.04 \\
Power trunk & 2 & 2.54 & 0.53 & 5 & 0.01 \\
Day tail 9 & & & & & \\
Power & 1 & 3.61 & 0.52 & 3 & 0.31 \\
Power trunk & 2 & 3.51 & 0.48 & 2 & 0.18 \\
Day tail 11 & & & & & \\
Power & 1 & 3.13 & 0.41 & 4 & 0.20 \\
Power trunk & 2 & 3.01 & 0.59 & 3 & 0.15 \\
Day Ttail 13 & & & & & \\
Power & 1 & 3.26 & 0.45 & 4 & 0.47 \\
Power trunk & 2 & 3.15 & 0.55 & 3 & 0.19 \\
Day tail 15 & & & & & \\
Power & 1 & 2.93 & 0.58 & 4 & 0.61 \\
Power trunk & 2 & 2.88 & 0.42 & 3 & 0.39 \\
Day tail 17 & & & & & \\
Power & 1 & 2.71 & 0.39 & 4 & 0.67 \\
$\begin{array}{l}\text { Power trunk } \\
\text { Night tail 19 }\end{array}$ & 2 & 2.65 & 0.61 & 3 & 0.48 \\
Power & 1 & 2.94 & 0.56 & 4 & 0.72 \\
Power trunk & 2 & 2.87 & 0.44 & 3 & 0.48 \\
Night tail 21 & & & & & \\
Power & 1 & 2.29 & 0.29 & 7 & 0.26 \\
Power trunk & 2 & 2.20 & 0.71 & 6 & 0.20 \\
Night tail 23 & & & & & \\
Power & 1 & 2.87 & 0.53 & 5 & 0.45 \\
Power trunk & 2 & 2.81 & 0.47 & 4 & 0.36 \\
\hline
\end{tabular}

square test always classifies this model as significantly different from the empirical distribution of step lengths (Table 2). Secondly, while the 2 power laws fail the goodness of fit test for half of the time intervals in the whole series (Table 2), the tail distribution is significantly different from the 2 models only at the interval between 06:00 and 08:00 h (Table 3). Thirdly, for most intervals the AIC weights did not discriminate much between the power law and its truncated version (Tables $2 \& 3$ ). Also, the AIC weights gave approximately the same result for the whole series and the tail. Since the power law has non-negligible probabilities for unrealistic long step length, we conclude that the truncated power law is the best model for the move-step distributions of $P$. periphylla. Since the truncated power law is (1) consistent with the distribution of the observed movesteps in the tail (chi-square test of goodness of fit to step lengths over $2.9 \mathrm{~m}$, Table 3) and (2) slightly favoured by the AIC (Table 3), we conclude that $P$. periphylla with Mode 4 behaviour performs a Lévylike flight in the darkest period. From the plot of the exponent versus the midpoint hour of the interval (Fig. 3), it is seen that the exponent goes through a diel cycle with values alternating significantly below 3 at night and in the darkest daytime period and significantly above 3 in the lightest part of the day. This shift in the exponent means that these P. periphylla perform a Lévy-like flight during the night and the darkest period of daytime.

The diel cycle in swimming behaviour was also reflected in the recorded swimming directions (Fig. 4). Records from the night-time portrayed swimming in both directions, while downward tracks prevailed throughout the daytime. Total numbers of tracks were higher during the daytime periods (Fig. 4).

\section{DISCUSSION}

Our analysis reveals 3 striking aspects of the vertical motility of Periphylla periphylla. The step lengths are highly skewed, the statistical analysis indicates that the truncated power law fits to the data at all times, and the frequency of long step lengths during the night is characteristic of a Lévy-flight distribution. The acoustic target tracking furthermore suggests changes in prevailing swimming direction between day and night, with strong predominance of descending tracks throughout the daytime. This latter finding may have been influenced by the method, as acoustic target tracking of $P$. periphylla tends to have a higher probability of detecting tracks during descent (Klevjer et al. 2009). Regardless, this metric also reflects the diel changes in individual swimming behaviour among $P$. periphylla.

It should be emphasized that whether the step length distribution follows a power law or not, the essential feature is that about $4 / 5$ of the swimming distances are less than $2.9 \mathrm{~m}$ and a few displacements are extremely long with a maximum of $85 \mathrm{~m}$. This pattern allows us to categorize the night observations as Lévy-flight (Bartumeus et al. 2002, Travis 

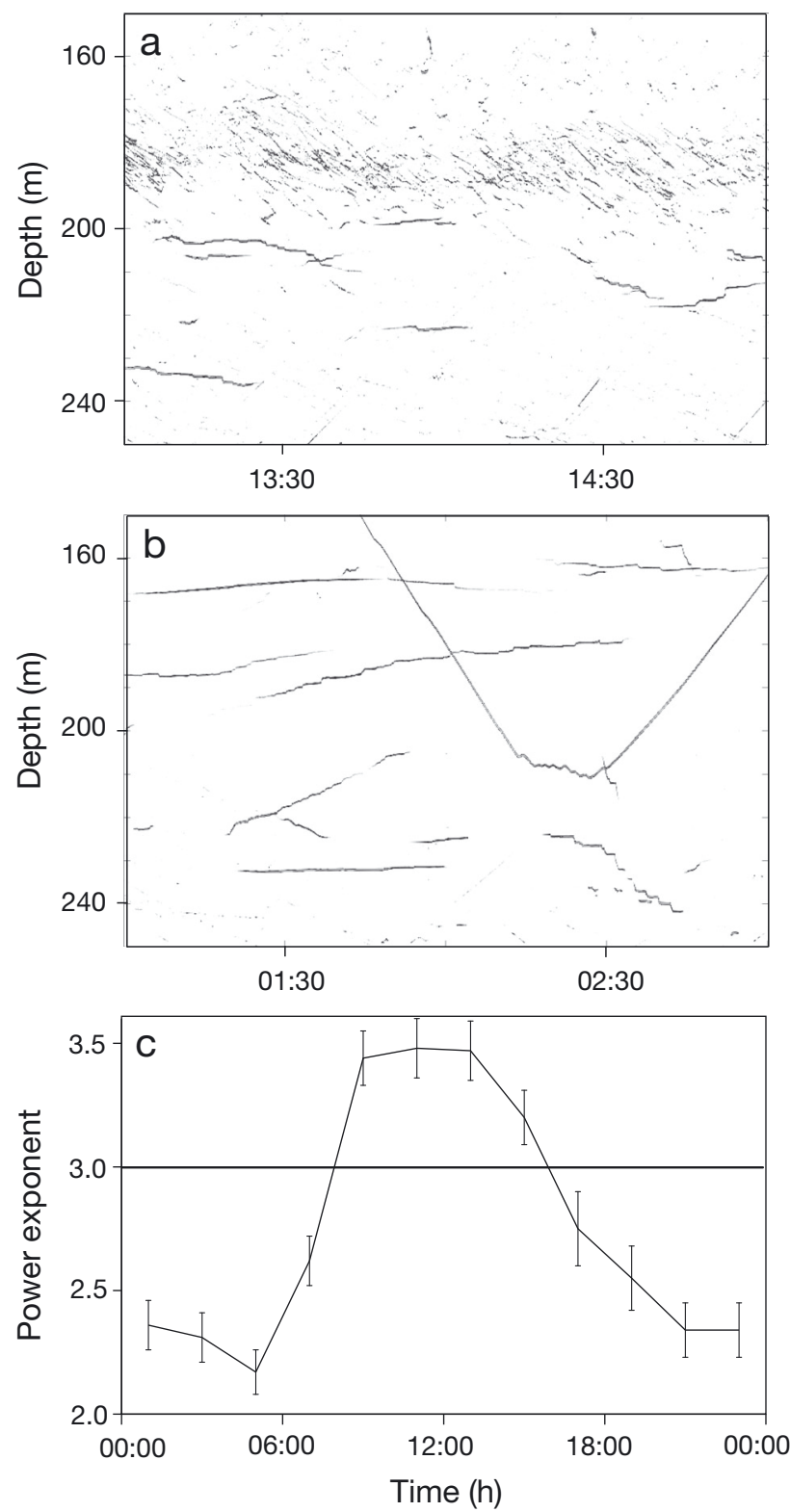

Fig. 3. (a,b) Two echograms illustrating typical behaviour of Periphylla periphylla during the (a) day and (b) night. The jellyfish can be seen as long traces on the echograms, which both span $100 \mathrm{~m}$ and $2 \mathrm{~h}$ in time. Note that only the $y$-axis represents jellyfish movements, as movements of echo traces along the $x$-axis represent progressing time. (c) $24 \mathrm{~h}$ cycle in the swimming behaviour of $P$. periphylla (compiled data from $3 \mathrm{wk}$ ) as characterized by the estimated values of the truncated power law exponent $(\alpha)$. The estimates of $\alpha$ and the corresponding $95 \%$ confidence intervals are the maximum likelihood estimators. All 12341 observed swimming tracks were classified into 12 discrete time intervals of $2 \mathrm{~h}$, the first from 0:00 to 2:00 $\mathrm{h}$ and the exponent values are plotted at the midpoint hours. Values below the horizontal line with $\alpha=3$ represent a Lévy-like flight. Times for sunrise and sunset were $08: 40$ and 14:54 h UTC for start of the observation period (10 Jan 2007) and 08:03 and 15:43 h UTC at end of the observation period (30 Jan)
2007). Short displacements are an efficient way of exploiting resources nearby, and it is combined with some long displacements in order to discover resources further away.

Our application of recommended statistical tools (Johnson \& Omland 2004, Clauset et al. 2007, Edwards et al. 2007, Edwards 2008) indicates that the truncated power law provides the best model for the jellyfish swimming behaviour. Using a large data set on vertical search among different marine predators, Sims et al. (2008) concluded that 'Lévy-like' behaviour is widespread but that animals often undertake other behaviours, such as social interactions or predator avoidance, which may weaken the Lévy signal. In contrast, Edwards (2008) concluded that Lévy flights are not as common as previously thought, and Edwards et al. (2007) argued that many published results could be ascribed to artefacts due to small and biased data sets. Gautestad \& Mysterud (2013) showed that if the time interval between 2 successive observations of position is large (they used GPS data on red deer), a true underlying Lévy flight may erroneously be interpreted as a Brownian motion. However, since our echo sounder scanned the water column once per second, this possible bias is absent in our study (see also Gautestad 2013).

The Lévy-like search pattern is likely to increase the foraging efficiency of $P$. periphylla during the night. But why is such a pattern not observed during the daytime? Lévy-type movements presumably maximize the probability that jellyfish encounter sparsely distributed, high density micropatches of prey (Hays et al. 2012). The fine-scale vertical distribution of potential prey in the depth of Lurefjorden is not known; hence we cannot assess the importance for prey search of any alternating strata of high and low densities of prey. Yet there is no reason to assume increased micro patchiness at night. It might be speculated that the prey is better at escaping from the tactile predator in the presence of light and that $P$. periphylla has evolved reduced daytime activity. It is noteworthy that the exponent of the power laws reaches the lowest value at the darkest period of the night. However, the 'jellyfish fjord' is a curious marine system as the density of likely prey is much higher than in the open sea and the potential competition/predation by fish is greatly reduced (Fosså 1992, Mills 1995, Bagøien et al. 2001). Since the apparently high zooplankton abundance violates the standard assumption about the prey field used in the derivation of the optimality of Lévy flights, we were in fact surprised to observe Lévy-like flights. 


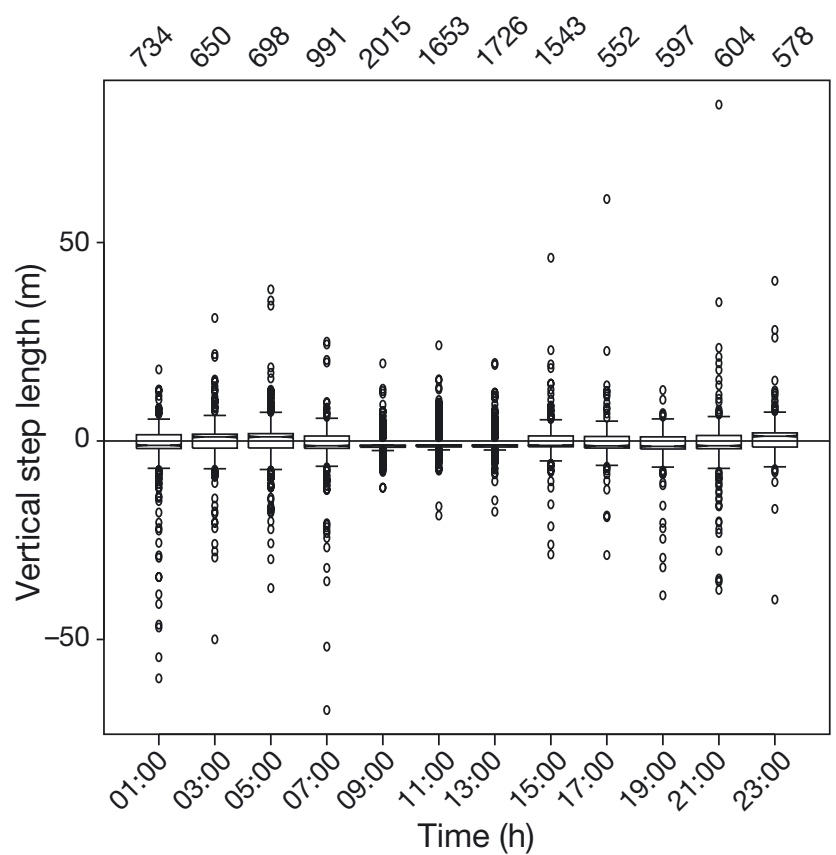

Fig. 4. Diel patterns in vertical step lengths of Periphylla periphylla. Positive values refer to upward swimming, negative to downward swimming. The boxes depict the median (horizontal line) and the 25 and 75 percentiles; whiskers refer to 1.5 times the interquartile range and dots represent measurements outside of this range. Total numbers of registrations for each time period are given at top of figure. Times for sunrise and sunset were 08:40 and 14:54 h UTC for start of the observation period (10 Jan 2007) and 08:03 and 15:43 h UTC at end of the observation period (30 Jan)

Hays et al. (2012) reported that the jellyfish Rhizostoma octopus exhibits movement patterns approximated by Lévy-like flights in a water column of less than $20 \mathrm{~m}$ and associated this behaviour with prey search. Similar search patterns were also ascribed to the jellyfishes Cyanea capillata and Phacellophora camtschatica by Moriarty et al. (2012). Our data for Periphylla periphylla indicate that the ability to perform Lévy-like flights is more widespread among jellyfish species than previously thought. Sørnes \& Aksnes (2004) demonstrated in feeding experiments that what appears to be a high prey concentration for a visual predator might appear low for a tactile predator. An intuitive hypothesis is that the adaptive value of performing Lévy-like flights is to increase the efficiency of prey search in a stochastic environment (Sims et al. 2008). However, this hypothesis does not imply that jellyfish compete strongly with zooplanktivorous fish for plankton prey.

Tactile feeding is independent of light per se and $P$. periphylla appears to be adapted to the dark environment of the deep open sea. Also, coastal locations that have been invaded appear to be dark because of high light attenuation of the coastal water combined with relatively deep fjord basins (Eiane et al. 1999, Aksnes et al. 2004, Sørnes et al. 2007). It has been suggested that the $P$. periphylla mass occurrences, combined with the absence of fish, have developed because of a gradual increase in the light attenuation of the Norwegian coastal water (Eiane et al. 1999, Sørnes et al. 2007, Aksnes et al. 2009). Thus, these locations appear to have obtained the darkness of the deep ocean and therefore offer a suitable habitat for Periphylla, but are less suitable for visually searching fishes. A similar shift in the competitive relationship between fish and jellyfish has also been hypothesized for the Baltic Sea (Haraldsson et al. 2012). A trend of increasing light attenuation (Dupont \& Aksnes 2013) is expected to continue in the coming years as a result of a climate change-associated increase in coloured dissolved organic matter (CDOM) of terrestrial origin (Larsen et al. 2011) that enters coastal waters.

The Lévy-flight foraging hypothesis predicts that the optimal search strategy is a Lévy flight when the prey is sparse and unpredictably distributed (Viswanathan et al. 1999). In contrast, a Brownian random walk suffices when foraging on abundant prey. Humphries et al. (2010) found strong support for this hypothesis for open-ocean predatory fish (sharks, tuna, billfish and ocean sunfish). However, our data on $P$. periphylla might question the generality of the Lévy-flight foraging hypothesis, because one may argue that a Lévy flight is not expected in a resourcerich environment, owing to a relatively high frequency of interrupts on path directionality (Gautestad \& Mysterud 2013). Thus, according to foraging theory, the high zooplankton concentration of Lurefjorden (Eiane et al. 1999, Bagøien et al. 2001) should rather induce a Brownian random walk in $P$. periphylla. It is possible that the Lévy-like search pattern reported here has evolved in the food scarce oceanic environment, which is the main habitat of this jellyfish and also the likely source for the population in Lurefjorden. Alternatively, the large individuals we have addressed in this study may forage on different and scarcer prey sources than the abundant zooplankton.

Acknowledgements. Anders Røstad was instrumental during the acoustic studies and he also provided Fig. 1.

\section{LITERATURE CITED}

Aksnes DL, Nejstgaard J, Sædberg E, Sørnes T (2004) Optical control of fish and zooplankton populations. Limnol Oceanogr 49:233-238

Aksnes DL, Dupont N, Staby A, Fiksen Ø, Kaartvedt S, Aure 
J (2009) Coastal water darkening and implications for mesopelagic regime shifts in Norwegian fjords. Mar Ecol Prog Ser 387:39-49

Bagøien E, Kaartvedt S, Aksnes DL (2001) Vertical distribution and mortality of overwintering Calanus. Limnol Oceanogr 46:1494-1510

Balk H, Lindem T (2000) Improved fish detection in data from split-beam sonar. Aquat Living Resour 13:297-303

Bartumeus F, Catalan J, Fulco UL, Lyra ML, Viswanathan GM (2002) Optimizing the encounter rate in biological interactions: Lévy vs. Brownian strategies. Phys Rev Lett 88:097901

Brede R, Kristensen FH, Solli H, Ona E (1990) Target tracking with a split-beam echo sounder. Rapp P-V Reùn Cons Int Explor Mer 189:254-263

Clauset A, Shalizi CR, Newman MEJ (2007) Power-law distributions in empirical data. SIAM Rev 51:661-703

Dalpadado P, Ellertsen B, Melle W (1998) Summer distribution patterns and biomass estimates of macrozooplankton and micronekton in the Nordic Seas. Sarsia 83: 103-116

> Dupont N, Aksnes DL (2013) Centennial changes in water clarity of the Baltic Sea and the North Sea. Estuar Coast Shelf Sci 131:282-289

$>$ Dupont N, Klevjer TA, Kaartvedt S, Aksnes DL (2009) Diel vertical migration of the deep-water jellyfish Periphylla periphylla simulated as individual responses to absolute light intensity. Limnol Oceanogr 54:1765-1775

Edwards AM (2008) Using likelihood to test for Lévy flight search patterns and for general power-law distributions in nature. J Anim Ecol 77:1212-1222

Edwards AM, Phillips RA, Watkins NW, Freeman MP and others (2007) Revisiting Lévy flight search patterns of wandering albatrosses, bumblebees and deer. Nature 449:1044-1048

Eiane K, Aksnes DL, Bagøien E, Kaartvedt S (1999) Fish or jellies - a question of visibility? Limnol Oceanogr 44: $1352-1357$

Fosså JH (1992) Mass occurrence of Periphylla periphylla (Schypozoa, Coronatae) in a Norwegian fjord. Sarsia 77 : $237-251$

Gautestad AO (2013) Lévy meets Poisson: a statistical artifact may lead to erroneous re-categorization of Lévy walk as Brownian motion. Am Nat 181:440-450

Gautestad AO, Mysterud A (2013) The Lévy flight foraging hypothesis: Forgetting about memory may lead to false verification of Brownian motion. Movement Ecol 1:9

> Haraldsson M, Tönnesson $\mathrm{K}$, Tiselius $\mathrm{P}$, Thingstad $\mathrm{TF}$, Aksnes DL (2012) Relationship between fish and jellyfish as a function of eutrophication and water clarity. Mar Ecol Prog Ser 471:73-85

> Hays GC, Kennedy H, Frost BW (2001) Individual variability in diel vertical migration in a marine copepod: why some individuals remain at depth when others migrate. Limnol Oceanogr 46:2050-2054

> Hays GC, Bastian T, Doyle TK, Fossette S and others (2012) High activity and Lévy searches: jellyfish can search the water column like fish. Proc R Soc B 279:465-473

Humphries NE, Queiroz N, Dyer JRM, Pade NG and others (2010) Environmental context explains Lévy and Brownian movement patterns of marine predators. Nature 465:

Editorial responsibility: Marsh Youngbluth,

Fort Pierce, Florida, USA
1066-1069

Jarms G, Tiemann H, Båmstedt U (2002) Development and biology of Periphylla periphylla (Schypozoa, Coronatae) in a Norwegian fjord. Mar Biol 141:647-657

> Johnson JB, Omland KS (2004) Model selection in ecology and evolution. Trends Ecol Evol 19:101-108

> Kaartvedt S, Klevjer TA, Torgersen T, Sornes TA, Rostad A (2007) Diel vertical migration of individual jellyfish (Periphylla periphylla). Limnol Oceanogr 52:975-983

Kaartvedt S, Titelman J, Røstad A, Klevjer TA (2011) Beyond the average: diverse individual migration patterns in a population of mesopelagic jellyfish. Limnol Oceanogr 56: 2189-2199

Klevjer TA, Kaartvedt S, Båmstedt U (2009) In situ behaviour and acoustic properties of the deep living jellyfish Periphylla periphylla. J Plankton Res 31:793-803

> Larsen S, Andersen T, Hessen DO (2011) Climate change predicted to cause severe increase of organic carbon in lakes. Glob Change Biol 17:1186-1192

> Mills CE (1995) Medusae, siphonophores and ctenophores as planktivorous predators in changing global ecosystems. ICES J Mar Sci 52:575-581

Mills CE (2001) Jellyfish blooms: are populations increasing globally in response to changing ocean conditions? Hydrobiologia 451:55-68

Moriarty PE, Andrews KS, Harvey CJ, Kawase M (2012) Vertical and horizontal movement patterns of scyphozoan jellyfish in a fjord-like estuary. Mar Ecol Prog Ser 455:1-12

Purcell JE, Uye S, Lo WT (2007) Anthropogenic causes of jellyfish blooms and their direct consequences for humans: a review. Mar Ecol Prog Ser 350:153-174

> Richardson AJ, Bakun A, Hays GC, Gibbons MJ (2009) The jellyfish joyride: causes, consequences and management responses to a more gelatinous future. Trends Ecol Evol 24:312-322

> Sims DW, Southall EJ, Humphries NE, Hays GC and others (2008) Scaling laws of marine predator search behaviour. Nature 451:1098-1102

> Sørnes TA, Aksnes DL (2004) Predation efficiency in visual and tactile predators. Limnol Oceanogr 49:69-75

Sørnes TA, Aksnes DL, Båmstedt U, Youngbluth MJ (2007) Causes for mass occurrences of the jellyfish, Periphylla periphylla; an hypothesis that involves optically conditioned retention. J Plankton Res 29:157-167

> Sørnes TA, Hosia A, Båmstedt U, Aksnes DL (2008) Swimming and feeding in Periphylla periphylla (Schypozoa, Coronatae). Mar Biol 153:653-659

Tiemann H, Sötje I, Johnston BD, Flood PR, Båmstedt U (2009) Documentation of potential courtship behaviour in Periphylla periphylla (Cnidaria: Scyphozoa). J Mar Biol Assoc UK 89:63-66

- Travis J (2007) Do wandering albatrosses care about math? Science 318:742-743

> Viswanathan GM, Buldyrev SV, Havlin S, da Luzk MGE, Raposok EP, Stanley HE (1999) Optimizing the success of random searches. Nature 401:911-914

> Youngbluth M, Båmstedt U (2001) Distribution, abundance, behavior and metabolism of Periphylla periphylla, a mesopelagic coronate medusa in a Norwegian fjord. Hydrobiologia 451:321-333

Submitted: January 9, 2014; Accepted: July 10, 2014 Proofs received from author(s): September 29, 2014 